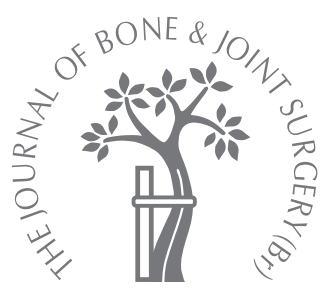

A. I. Tsirikos, M. J. McMaster

From The Royal Hospital for Sick Children, Edinburgh, Scotland

A. I. Tsirikos, PhD, MD, FRCS, Consultant Orthopaedic and Spine Surgeon

M. J. McMaster, MD, FRCS, Honorary Consultant Orthopaedic and Spine Surgeon

Scottish National Spine Deformity Centre Royal Hospital for Sick Children, Sciennes Road, Edinburgh EH9 1LF, UK.

Correspondence should be sent to $\mathrm{Mr}$ A. I. Tsirikos; e-mail: atsirikos@hotmail.com

(C)2010 British Editorial Society of Bone and Joint Surgery doi:10.1302/0301-620X.92B3. $23075 \$ 2.00$

$J$ Bone Joint Surg $[\mathrm{Br}]$ 2010;92-B:430-5.

Received 10 July 2009;

Accepted after revision 29

October 2009

\title{
Infantile developmental thoracolumbar kyphosis with segmental subluxation of the spine
}

We report five children who presented at the mean age of 1.5 years (1.1 to 1.9) with a progressive thoracolumbar kyphosis associated with segmental instability and subluxation of the spine at the level above an anteriorly-wedged hypoplastic vertebra at L1 or L2. The spinal deformity appeared to be developmental and not congenital in origin. The anterior wedging of the vertebra may have been secondary to localised segmental instability and subsequent kyphotic deformity.

We suggest the term 'infantile developmental thoracolumbar kyphosis with segmental subluxation of the spine' to differentiate this type of deformity from congenital displacement of the spine in which the congenital vertebral anomaly does not resolve. Infantile developmental kyphosis with segmental subluxation of the spine, if progressive, may carry the risk of neurological compromise. In all of our patients the kyphotic deformity progressed over a period of three months and all were treated by localised posterior spinal fusion. At a mean follow-up of 6.6 years (5.0 to 9.0), gradual correction of the kyphosis was seen on serial radiographs as well as reconstitution of the hypoplastic wedged vertebra to normality. Exploration of the arthrodesis was necessary at nine months in one patient who developed a pseudarthrosis.

Before they begin to walk normal infants have a long, smooth kyphotic posture from the occiput to the sacrum. ${ }^{1}$ Once they gain head and neck control they slowly develop a cervical lordosis. The normal lumbar lordosis does not appear until the child is able to stand and walk. The spine at the thoracolumbar junction is flat in the sagittal plane. ${ }^{1}$

The development of a thoracolumbar kyphosis in walking infants and young children may be due to spinal injury, infection, neoplasm, skeletal dysplasia, or a congenital vertebral anomaly. ${ }^{2}$ In non-walking infants and young children, the primary cause of a thoracolumbar kyphosis is a myelomeningocele usually with a high-level neurological deficit. $^{3}$

Most patients with a congenital kyphosis or kyphoscoliosis have a well-aligned spinal canal. ${ }^{4}$ In a small group with congenital kyphosis due to an anterior failure of vertebral body formation, the spinal canal is not only sharply angulated but also subluxed or dislocated at the apex of the deformity. ${ }^{5-7} \mathrm{Seg}-$ mental subluxation or dislocation of the spine has been described as potentially the most serious form of congenital kyphosis, even though not all the patients included in the initial reports had associated congenital vertebral abnormalities. ${ }^{5,6}$

Campos et $\mathrm{al}^{2}$ reported seven otherwise apparently normal children who presented with what they described as an infantile thoracolumbar kyphosis associated with single-level hypoplasia of the anterior aspect of the body of a lumbar vertebra. They noted that with time there was a spontaneous gradual correction of the kyphosis, raising the question of the effect of mechanical overload on the vertebral body and its resolution as the child developed an erect posture.

We report five children with a progressive thoracolumbar kyphosis and segmental subluxation of the spine occurring above a hypoplastic, anteriorly-wedged vertebra. These patients were thought initially to represent a variant of congenital kyphosis with displacement in the sagittal plane. However, results of surgical treatment suggest that this group of patients should not be classified as having a congenital kyphosis because the apparent congenital vertebral anomaly resolved to normality. This may represent a progressive type of infantile developmental kyphosis with associated segmental vertebral subluxation occurring at the junction between the rigid thoracic 
and more mobile lumbar spine, thereby causing secondary anterior wedging of the vertebra.

\section{Patients and Methods}

We reviewed the medical records and spinal radiographs of 750 consecutive patients who were diagnosed with a congenital spinal deformity at our institution between 1960 and 2008. Of these, $589(79 \%)$ had a scoliosis, $106(14 \%)$ a kyphoscoliosis and $55(7 \%)$ a pure kyphosis. Patients who had myelomeningocele, Scheuermann's disease, neurofibromatosis, skeletal dysplasia, infection, trauma or a neoplasm were excluded, as the aim was to identify patients with congenital vertebral displacement.

We identified five boys who had a thoracolumbar kyphosis with associated vertebral displacement and a 'step-off' deformity occurring above a hypoplastic anteriorly-wedged vertebra. The vertebral anomalies were detected on anteroposterior and lateral radiographs. The degree of spinal deformity in the coronal and sagittal planes was measured using the modified Cobb method on serial spinal radiographs taken with the patient standing. ${ }^{8,9}$ The Meyerding method was used to measure the degree of vertebral subluxation in the sagittal plane. ${ }^{10}$ Care was taken to measure all the curves using the same anatomical landmarks on the serial spinal radiographs taken before the surgery and during follow-up.

All five patients had a whole spinal MR scan, as well as cardiac and renal assessment. Their mean age at presentation was 1.5 years (1.1 to 1.9 ). They were referred because the parents had noted a prominence in their child's spine. All five were neurologically normal and walked independently. None complained of back pain. On clinical examination, there was a considerable thoracolumbar kyphotic deformity, which partially corrected in the supine position.

On radiological examination, the level of the vertebral subluxation was at T12-L1 in four patients and at L1-2 in one. The anterior subluxation and 'step-off' deformity occurred only in the sagittal plane and was at the level above an anomalous vertebra (Figs 1 and 2). The anomalous vertebra at L1 (four patients) and L2 (one patient) was hypoplastic with anterior wedging and an indentation in the anterosuperior aspect of the vertebral body producing a beaked appearance. The posterior elements were normal. The wedged vertebra did not sublux posteriorly into the spinal canal and the spine was well aligned both above and below to the level of the displacement. There was no rotatory or transverse displacement and no evidence of scoliosis above or below the kyphotic segment.

MRI showed a normal appearance of the pedicles and facet joints bilaterally at the level of the anomalous vertebra in all five patients. Because of the young age of the patients and poor compliance, a supine lateral spinal radiograph in hyperextension against a bolster to assess flexibility of the deformity was not taken. However, MRI showed the kyphosis and segmental vertebral displacement to be flexible and partially correctable in the supine position (Figs 1 and 2). There were no intraspinal anomalies.
Cardiac and renal investigations were normal. None of the patients had Sprengel's shoulder, Klippel-Feil syndrome or anomalies of the ribs and chest wall. There were no medical comorbidities.

Operative treatment. The indication for surgical treatment was progression of the kyphotic deformity of $10^{\circ}$ to $15^{\circ}$ in all five patients over a period of three months between the initial diagnosis and decision to operate. As they were all walking, there was concern that compression of the spinal cord could develop at the site of displacement.

All five patients were treated by a localised posterior spinal arthrodesis using allograft bone without instrumentation, extending two disc levels above and below the anomalous vertebra. The patients were considered too young to have donor bone removed from their iliac crest, which otherwise would have been preferable. At operation, the posterior elements of the wedged vertebra and the facet joints above and below were found to be normal. A moulded underarm spinal jacket was applied postoperatively, with the patient under general anaesthesia and lying supine on a plaster frame. The spinal jacket was maintained for three months.

\section{Results}

Pre-operatively, the mean kyphosis was $45^{\circ}\left(35^{\circ}\right.$ to $\left.50^{\circ}\right)$ which was reduced post-operatively after application of the spinal jacket to a mean of $24^{\circ}\left(10^{\circ}\right.$ to $\left.32^{\circ}\right)$. However, the mean percentage segmental vertebral subluxation and stepoff deformity remained unchanged at $52 \%$ (30\% to $70 \%)$.

Post-operatively, all five patients were followed by serial spinal radiographs with the child standing. In one patient, after removal of the plaster jacket and over a period of six months, there was progression of the kyphosis from $32^{\circ}$ to $43^{\circ}$, but with no change in the degree of vertebral displacement, which remained at $70 \%$. Exploration nine months after the initial operation showed a pseudarthrosis, which was treated by regrafting using autologous rib graft. Repeat exploration of the fusion mass six months later confirmed solid fusion.

At the latest follow-up, at a mean of 6.6 years (5.0 to 9.0) after the initial operation, none of the children had a clinical deformity or spinal symptoms. They were all taking part in normal activities, including sport. Serial radiographs taken during the follow-up period showed gradual correction of the segmental kyphosis and vertebral subluxation as well as reconstitution of the previously hypoplastic vertebral body to normality. This occurred mainly in the first two years (Figs 1 and 2). In all patients the spine showed normal coronal and sagittal balance.

\section{Discussion}

Our patients were thought initially to have a congenital kyphosis because of partial anterior failure of vertebral formation with an associated segmental displacement of the spine and malalignment of the spinal canal with a step-off deformity in the sagittal plane. The initial diagnosis was 


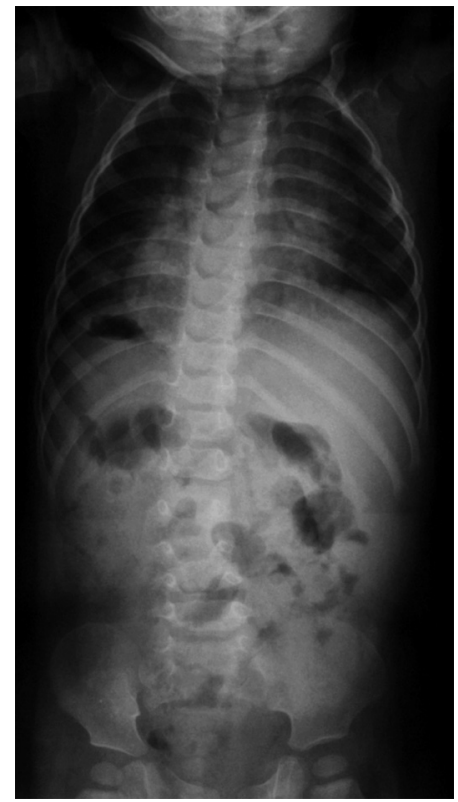

Fig. 1a

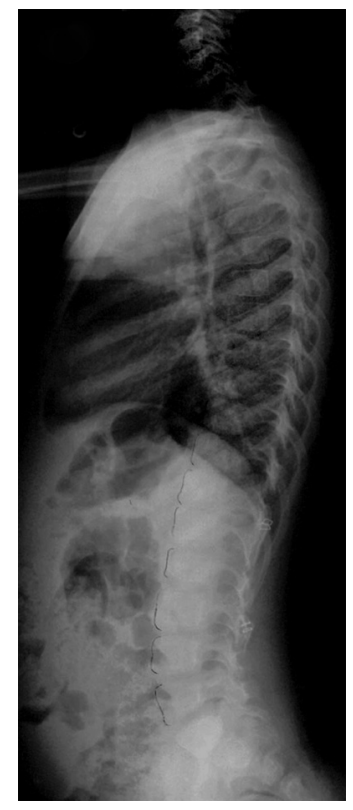

Fig. 1c

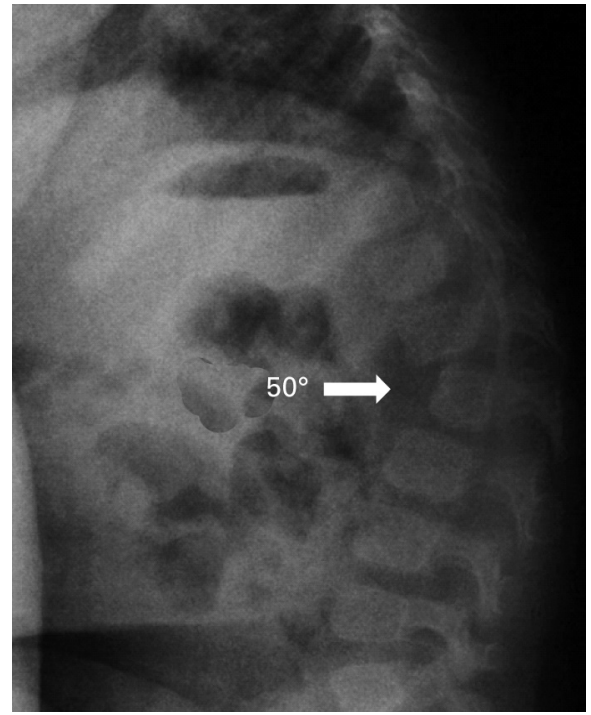

Fig. $1 \mathrm{~b}$

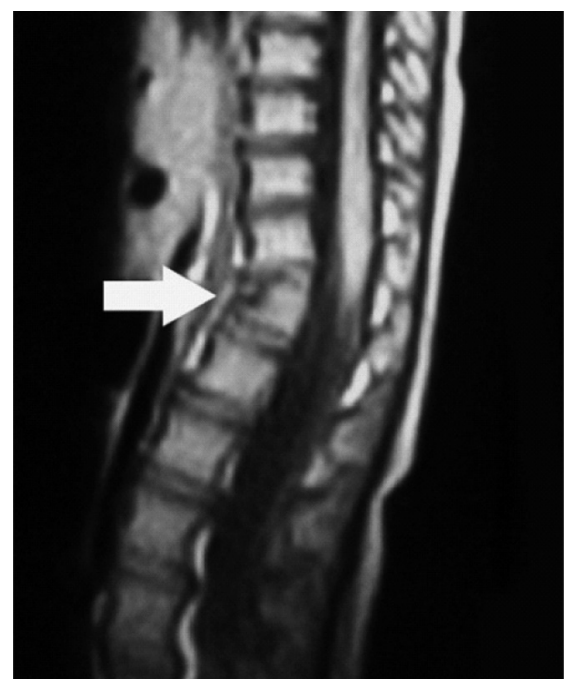

Fig. 1d

Figure 1a - Pre-operative anteroposterior radiograph of the spine at the age of 1 year 2 months showing normal spinal balance. Figure $1 \mathrm{~b}$ Pre-operative lateral radiograph of the spine showing a hypoplastic anteriorly wedged vertebra at $L 2$ with anterior vertebral subluxation at the disc level above and an associated kyphosis of $50^{\circ}$. Figure $1 \mathrm{c}$ - Lateral radiograph of the spine at follow-up aged 4 years 7 months showing normal sagittal balance of the spine and complete reconstitution of the anomalous vertebra to normal. Figure 1d - Pre-operative MRI scan of the spine showing the segmental kyphosis, which is partially correctable in the supine position, as well as the hypoplastic vertebra (white arrow). Note the significant difference on the sagittal alignment of the thoracolumbar/lumbar spine between the sitting lateral radiograph (b) and the supine MRI scan (d), which confirms the presence of severe segmental instability.

based on the radiological appearance of the anomalous, anteriorly-wedged and beaked vertebra which resembled a congenital posterior wedged vertebra. This was thought to have resulted in segmental instability and subluxation of the spine at the level above the hypoplastic vertebra, which was at the apex of the kyphosis. All our patients had normal neurological function with an intact posterior vertebral arch and normal facet joints. Despite the beaked appearance of 


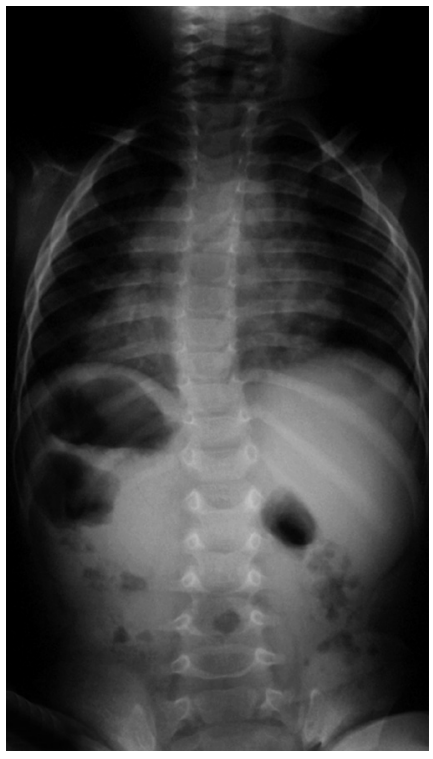

Fig. $2 a$

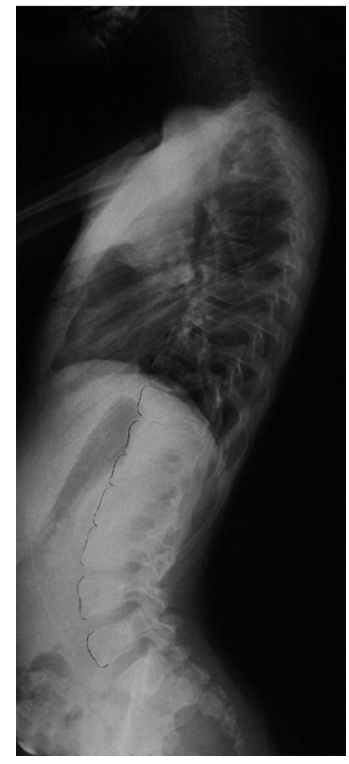

Fig. 2c

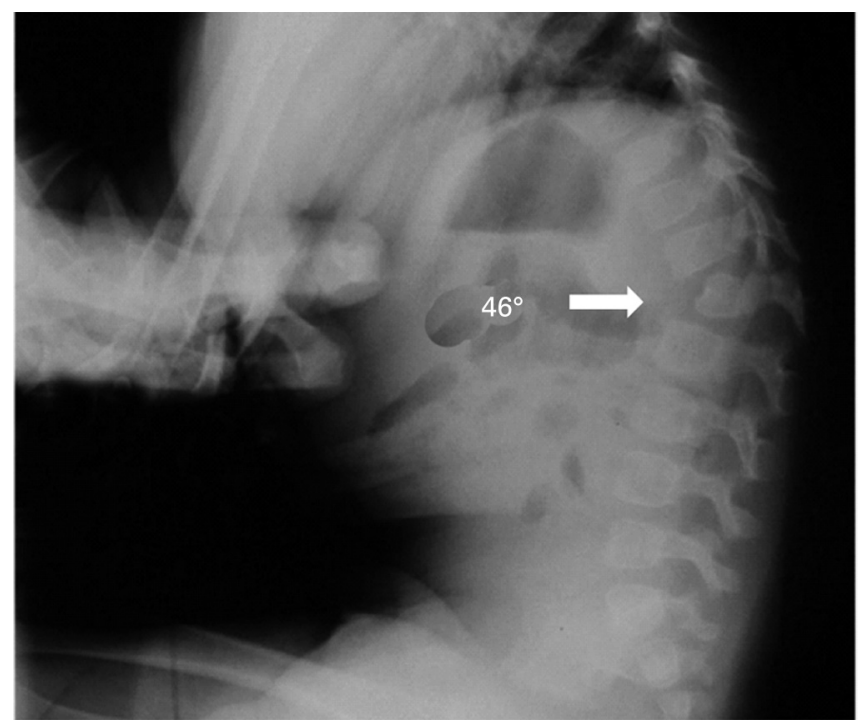

Fig. 2b

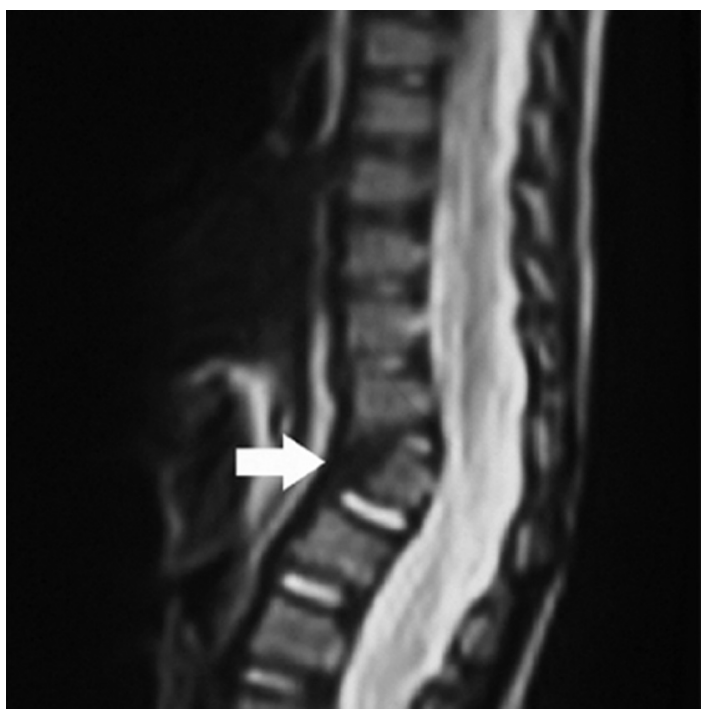

Fig. 2d

Pre-operative radiograph of the spine at the age of 1 year 3 months showing (a) a normal spinal balance, (b) a hypoplastic anteriorly-wedged vertebra at L1 with anterior vertebral subluxation occurring at the T12-L1 level and an associated thoracolumbar kyphosis measuring $46^{\circ}$, (c) lateral radiograph of the spine at follow-up at the age of seven years five months showing normal sagittal balance of the spine and complete reconstitution of the anomalous vertebra and (d) Pre-operative MR scan of the spine showing the segmental kyphosis, which is partially correctable in the supine position as well as the hypoplastic vertebra (white arrow). There is a considerable difference in the sagittal alignment of the thoracolumbar spine between the sitting lateral radiograph (b) and the supine MR scan (d) which confirms the presence of severe segmental instability.

the abnormal vertebra at the apex of the kyphosis, which can also be seen in patients with achondroplasia, ${ }^{11,12}$ there were no other clinical or radiological signs suggestive of skeletal dysplasia. MRI showed no intraspinal anomalies.

Congenital kyphosis and kyphoscoliosis is an uncommon deformity in which there is an abnormal posterior convex angulation of a segment of the spine due to developmental vertebral anomalies that impair longitudinal growth anterior or anterolateral to the transverse axis of vertebral rotation in the sagittal plane. ${ }^{9,13}$ These vertebral anomalies may be due to either a defect of formation or segmentation of one or more vertebral bodies occurring anteriorly or anterolaterally. 
Dubousset ${ }^{4}$ classified anterior defects of vertebral body formation resulting in a kyphosis or kyphoscoliosis into two types depending on the alignment of the spinal canal. In type 1 there is a partial failure of vertebral body formation with a well-aligned spinal canal and an intact posterior arch. In type 2 there is a dislocated canal which produces a step-off deformity in the sagittal plane and a bayonet-type appearance in the coronal plane, as well as abnormal posterior elements. The loss of continuity of the posterior margins of the adjacent vertebral bodies at the apex of the kyphosis has been designated as the 'step-off' sign which is the hallmark of a congenitally dislocated spine. ${ }^{4,6}$ However, in their original reports of rotatory dislocation of the spine, Duval-Beaupere and Dubousset ${ }^{5}$ and Dubousset et $\mathrm{al}^{6}$ included seven patients with skeletal dysplasias, five with neurofibromatosis, one with idiopathic scoliosis and only five with congenital vertebral anomalies.

In 1993, Shapiro and Herring ${ }^{7}$ used the term 'congenital vertebral displacement' to describe ten patients with a congenital kyphosis and an abrupt displacement of the spinal canal occurring above an anomalous vertebra which was at the apex of the deformity. They differentiated two patterns of deformity. Type A was more common and consisted of vertebral displacement occurring only in the sagittal plane as a result of a posterior hemivertebra. Type B affected all three planes and resulted in a rotatory, transverse and sagittal displacement, very similar to that of congenital dislocation of the spine as described by Dubousset et al. ${ }^{6}$

We thought initially that the vertebral abnormality and type of deformity in our patients represented a variant of type-A vertebral displacement. ${ }^{3}$ However, there were significant differences. With type-A displacement, the anomalous vertebra is a posterior hemivertebra and the deformity is potentially unstable because of a deficient posterior neural arch at the level of the abnormal vertebra and a tendency for the posterior hemivertebra to displace backwards into the spinal canal. This can result in either an acute paralysis after minor trauma or a gradually developing paraparesis as the deformity progresses. Also, intraspinal or systemic anomalies may be present.

In our group of patients with an angular kyphosis, the displacement also occurred in the sagittal plane above an anteriorly-wedged vertebra with a 'step-off' deformity. However, the spine was well aligned above and below the level of subluxation and the hypoplastic anteriorly-wedged vertebra was stable with no displacement posteriorly into the spinal canal. The posterior vertebral elements and pedicles were normal and we did not find any intraspinal abnormalities. All our patients showed progression of their deformity before operative treatment and although they had normal neurology there was concern that increasing deformity could cause compression of the spinal cord and neurological complications.

We found that early posterior spinal arthrodesis, in the presence of continuing anterior vertebral growth, allowed not only gradual spontaneous correction of the kyphosis but also complete reduction of the associated vertebral subluxation (Figs 1 and 2). There was also complete reconstitution of the anomalous wedged vertebra to normality in all five patients which occurred mainly in the first two years after posterior spinal arthrodesis. This finding suggests that the hypoplasia and anterior wedging of the vertebral body could be developmental in origin as a result of secondary disturbance of the anterior growth of the affected vertebra at the level of mechanical instability. The favourable clinical outcome after surgery may be an example of the HueterVolkmann principle in which the growth plates have increased growth in response to tension produced by the posterior fusion. ${ }^{14}$

Kim and Weinstein ${ }^{15}$ described two patients with an atypical form of congenital kyphosis with associated segmental vertebral displacement. The appearance of the anteriorly hypoplastic vertebra in their report was similar to that in our patients. However, it differed in that their patients had short pedicles and segmental narrowing of the spinal canal at the level of the displacement suggestive of a congenital abnormality. They performed a short anterior and a longer posterior spinal arthrodesis across the apex of the kyphosis. The kyphotic deformity in these two patients took much longer to correct when compared with our study. This may have been due to the short anterior spinal fusion limiting the potential for spontaneous anterior correction, which could only occur in the unfused levels above and below those which were included in the longer posterior fusion.

The absence of other abnormalities in the formation of the vertebral column and the neural structures apart from the anterior wedging of the hypoplastic vertebra differentiates the type of anomaly and associated deformity seen in our patients from congenital displacement of the spine. We therefore suggest that the term 'infantile developmental kyphosis with segmental subluxation of the spine' should be used to describe the condition seen in our five patients.

One of our patients developed a pseudarthrosis after posterior spinal arthrodesis with further progression of the kyphosis. Unfortunately, in young children it is not possible to assess the quality of a posterior spinal fusion simply on plain radiography. We therefore recommend exploration of the fusion mass if there is any loss of correction after removal of the spinal jacket following the initial arthrodesis to either repair a pseudarthrosis or augment a weak spinal fusion with additional autologous bone graft. In our patient, the pseudarthrosis was explored and repaired at nine months when the kyphosis had relapsed to $43^{\circ}$. The patient went on to develop a normal sagittal profile five years after the initial spinal procedure. Long-term followup to skeletal maturity will be required, even though surgery has been successful to date in all our patients.

Campos et $\mathrm{al}^{2}$ described seven infants with normal neurological function who developed a thoracolumbar kyphosis which was thought to have occurred secondary to lumbar hypoplasia affecting the L1 or L2 level. The appear- 
ance of the anomalous vertebra in their report was similar to that seen in our patients. The authors did not comment on the presence of segmental subluxation occurring at the level above the anteriorly wedge-shaped vertebra. However, Figure 1 in their article shows evidence of such subluxation. $^{4}$ The mean age at presentation was 5.3 months (birth to 11 months) and the mean angle of the kyphosis in sitting radiographs was $34.2^{\circ}\left(24^{\circ}\right.$ to $\left.41^{\circ}\right)$ at a mean age of 12 months. The mean kyphosis shown in the first standing radiograph was $19.7^{\circ}\left(10^{\circ}\right.$ to $\left.27^{\circ}\right)$. All the patients were treated by observation and the kyphosis gradually improved spontaneously. At a mean follow-up of 5.7 years, all the patients were asymptomatic and the radiological evaluation showed correction of the kyphosis to nearly normal.

The difference between these patients and our group is that all our patients presented later when they had started walking with a more severe and progressive thoracolumbar kyphosis, as well as segmental subluxation of the spine at the level above the anteriorly-wedged vertebra which we felt warranted surgical treatment. These two groups of patients may represent a spectrum of the same deformity in which some resolve spontaneously whereas others progress and require treatment. It is interesting that in both groups the kyphosis developed at the transitional thoracolumbar junction which suggests a mechanical cause for the condition. One weakness of our study is that in the presence of rapid kyphosis progression none of our patients were initially managed non-operatively. Because of the developmental nature of the deformity serial casting or bracing might have been effective.
Institutional review board approval was received in support of this study.

No benefits in any form have been received or will be received from a commercial party related directly or indirectly to the subject of this article.

\section{References}

1. Mac-Thiong J-M, Berthonnaud E, Dimar JR, Betz RR, Labelle H. Sagittal alignment of the spine and pelvis during growth. Spine 2004;29:1642-7.

2. Campos MA, Fernandes P, Dolan LA, et al. Infantile thoracolumbar kyphosis secondary to lumbar hypoplasia. J Bone Joint Surg [Am] 2008;90-A:1726-9.

3. Krajbich JI. Kyphectomy. In: Weinstein SL, ed. The pediatric spine: principles and practice. New York: Raven Press, 1994.

4. Dubousset J. Congenital kyphosis and lordosis. In: Weinstein SL, ed. The pediatric spine. New York: Raven Press, 1994:245-58.

5. Duval-Beaupere G, Dubousset J. Progressive rotational dislocation of the spine: mechanical process common to evolutive kyphoscoliosis complicated by neurologic disorder: a propos of 16 cases. Rev Chir Orthop Reparatrice Appar Mot 1972;58:32334 (in French).

6. Dubousset J, Duval-Beaupere G, Anquez L. Deformations vertebrales congenitales compliquees de troubles neurologiques dans compressions medullaires. In: Rougerie J, ed. Compressions medullaires. Paris: Masson, 1973:193-207.

7. Shapiro J, Herring J. Congenital vertebral displacement. J Bone Joint Surg [Am] 1993;75-A:656-62.

8. Cobb JR. Outline for the study of scoliosis. In: Instructional Course Lectures. American Academy of Orthopaedic Surgeons. Ann Arbor: JW Edwards, 1948:261-75.

9. McMaster MJ, Singh H. Natural history of congenital kyphosis and kyphoscoliosis: a study of 112 patients. J Bone Joint Surg [Am] 1999;81-A:1367-83.

10. Meyerding H. Low backache and sciatic pain associated with spondylolisthesis and protruded intervertebral disc: incidence, significance and treatment. J Bone Joint Surg 1941;23:461-70.

11. Levin TL, Berdon WE, Lachman RS, et al. Lumbar gibbus in storage diseases and bone dysplasias. Pediatr Radiol 1997;27:289-94.

12. Swischuk LE. The beaked, notched, or hooked vertebra: its significance in infants and young children. Radiology 1970;95:661-4.

13. McMaster MJ, Singh H. The surgical management of congenital kyphosis and kyphoscoliosis. Spine 2001;26:2146-55.

14. Hueter C. Anatomische studien an den extremitatengelenken neugebornener und erwachsener. Vischows Arch 1862;25:572 (in German).

15. Kim HW, Weinstein SL. Atypical congenital kyphosis: report of two cases with longterm follow-up. J Bone Joint Surg [Br] 1998;80-B:25-9. 